\title{
Habilidades empáticas na consulta de enfermagem ao cliente com doença cardiovascular: uma revisão integrativa
}

\section{Empathic skills on nursing consultation to the patient with cardiovascular disease: an integrative review}

\section{Habilidades empáticas en la consulta de enfermería al cliente con enfermedad cardiovascular: una revisión integradora}

Recebido: $13 / 11 / 2018$

Aprovado: 20/03/2019

Publicado: 13/05/2019
Diego da Silva Moreira ${ }^{1}$

Celia Caldeira Fonseca Kestenberg ${ }^{2}$ Priscila Cristina da Silva Thiengo ${ }^{3}$ Alexandre Vicente da Silva ${ }^{4}$ Elizabeth Rose Costa Martins ${ }^{5}$

O objetivo deste estudo é descrever as contribuições dos estudos científicos sobre consulta de enfermagem em nível ambulatorial, valorizando as habilidades empáticas, em clientes com doença cardiovascular. Foi realizada uma revisão integrativa com busca nas bases de dados da Biblioteca Virtual de Saúde em março de 2017, considerando o período de 2012 a 2016, sendo selecionados 15 artigos que deram origem a duas categorias: a Consulta de Enfermagem; e a avaliação dos clientes sobre as práticas do enfermeiro na Consulta de Enfermagem. Encontrou-se como resultados que a consulta de enfermagem deve ultrapassar os limites da orientação, proporcionando um real momento de sua transformação. Também que as habilidades empáticas como compaixão, escuta ativa e acolhedora, e a compreensão dos agravos à saúde, são fundamentais na construção da relação de confiança e na manutenção do vínculo terapêutico. Para tal, é fundamental que o profissional possua a compreensão abrangente das condições cardiovasculares, para implementar medidas efetivas. Destaca-se a necessidade de o enfermeiro atuar pensando no sujeito em todos os seus aspectos. Para isso, precisa possuir e ou desenvolver habilidades.

Descritores: Assistência Ambulatorial, Enfermagem cardiovascular; Empatia.

The aim of this study is to describe the contributions of scientific studies on nursing ambulatory care, valuing the empathic skills in clients with cardiovascular disease. An integrative review was carried out with search in the Virtual Health Library databases in March 2017, considering the period from 2012 to 2016, with 15 articles being selected, which gave rise to two categories: the Nursing Consultation; and evaluation of clients on nursing practice in nursing consultation. Regarding the results, it was found that the nursing consultation should exceed the limits of orientation, providing a real time of its transformation. In addition, the empathic skills like compassion, active and welcoming listening, and understanding of health problems, are fundamental in the trust building process and maintenance of the therapeutic relationship. For such, it is essential that the professional have the comprehensive understanding of cardiovascular conditions to implement effective measures. One highlights the need for nurses to act thinking about the subject in all aspects. To that end, they should have or develop skills.

Descriptors: Ambulatory care, Cardiovascular nursing; Empathy.

El objetivo de este estudio es describir las contribuciones de los estudios científicos sobre consulta de enfermería en nivel ambulatorial, valorando las habilidades empáticas, en clientes con enfermedad cardiovascular. Fue realizada una revisión integrativa con búsqueda en las bases de datos de la Biblioteca Virtual de Salud en marzo de 2017, considerando el periodo de 2012 a 2016, siendo seleccionados 15 artículos que dieron origen a dos categorías: Consulta de Enfermería: y la evaluación de los clientes sobre la práctica del enfermero en la Consulta de Enfermería. Se ha encontrado como resultados que la consulta de enfermería debe ultrapasar los límites de la orientación, proporcionando un real momento de su transformación. También que las habilidades empáticas como compasión, escucha activa y acogedora, y la compresión de los agravios a la salud, son fundamentales en la construcción de la relación de confianza y en la manutención del vínculo terapéutico. Para tal, es fundamental que el profesional posea la compresión abarcadora de las condiciones cardiovasculares, para implementar medidas efectivas. Se destaca la necesidad del enfermero actuar pensando en el sujeto en todos sus aspectos. Para eso, necesita poseer y o desarrollar habilidades.

Descriptores: Atención ambulatoria, Enfermería cardiovascular; Empatía.

1. Enfermeiro. Mestre em Enfermagem. Supervisor de Enfermagem da Policlínica Piquet Carneiro, Rio de Janeiro, RJ, Brasil. ORCID: 0000-0002-4292-0572 E-mail: diego_silvamoreira@hotmail.com

2. Enfermeira e Psicóloga Clínica. Doutora em Psicologia Social. Professora Adjunta da graduação e pós-graduação Latto Sensu da Faculdade de Enfermagem da Universidade Estadual do Rio de Janeiro (FE/UERJ), Rio de Janeiro, RJ, Brasil. ORCID: 0000-0001-9693-6791 E-mail: celiaprofuerj@gmail.com

3. Enfermeira. Mestre em Enfermagem. Professora Assistente da FE/UERJ, Rio de Janeiro, RJ, Brasil. ORCID: 0000-0003-0840-4838 E-mail: profprithiengo@gmail.com

4. Enfermeiro e Psicólogo. Mestre em Enfermagem. Professor Assistente da FE/UERJ, Rio de Janeiro, RJ, Brasil. 0RCID: 0000-0002-1240-0912

E-mail: alexvicentesilva@uol.com.br

5. Enfermeira. Doutora em Enfermagem. Professor Adjunto da FE/UERJ, Rio de Janeiro, RJ, Brasil. 0RCID: 0000-0001-5947-5535

E-mail: oigresrose@uol.com.br 


\section{INTRODUÇÃO}

$\mathbf{E}$ ste estudo tem como objeto a Consulta de Enfermagem em clientes com doença cardiovascular em nível ambulatorial: valorizando as habilidades empáticas.

Devido a hegemonia do modelo biomédico, tanto na maioria das escolas de formação profissional, quanto nas instituições hospitalares, os profissionais tendem a valorizar o tecnicismo durante a assistência, desconsiderando, na maioria das vezes, os aspectos individuais e emocionais do cliente ${ }^{1}$, fato que impacta negativa e diretamente na qualidade da assistência, pois impossibilita a realização do cuidado integral.

O sucesso do tratamento do cliente com doença cardiovascular (DCV) depende fortemente da participação e do envolvimento do usuário enquanto sujeito ativo de seu tratamento. Uma atitude de autocuidado que leve a estilos e práticas de vida mais saudáveis, assim como a adesão ao tratamento, não depende apenas de uma prescrição profissional, mas de uma conscientização do usuário sobre sua condição de saúde e a relação dela com suas práticas $^{2}$.

Para desenvolver a autonomia é necessário o desenvolvimento da educação em saúde com foco no autocuidado, aproximando o paciente de seu tratamento, tornando-o sujeito responsável por sua própria saúde 3 .

A Resolução COFEN 311/2007 diz que o profissional de enfermagem exerce suas atividades com competência para a promoção do ser humano na sua integralidade, de acordo com os princípios da ética e da bioética ${ }^{4}$.

Atualmente as DCVs são a causa mais frequente de mortalidade no mundo, tanto entre homens como em mulheres, produzindo um sério impacto funcional e emocional na vida de pacientes e familiares ${ }^{5}$. A discussão sobre as DCVs é de extrema relevância, pois, além do impacto sobre a qualidade de vida das pessoas, essas condições representam grandes despesas socioeconômicas para o país, pois são responsáveis por muitas internações, amputações, perdas de mobilidade e de outras funções neurológicas, as quais refletem em altos índices de absenteísmo, na redução da produtividade e no aumento das aposentadorias 6 .

No Brasil, no ano de 2015, estimou-se um custo direto e indireto com as DCVs de mais que $\mathrm{R} \$ 37$ bilhões, o que correspondia a 0,6\% do PIB (Produto Interno Bruto) e gasto per capita de $\mathrm{R} \$ 172,62^{7}$.

Assim, este estudo tem como objetivo descrever as contribuições dos estudos científicos sobre consulta de enfermagem em nível ambulatorial, valorizando as habilidades empáticas, em clientes com doença cardiovascular.

\section{METODO}

Trata-se de uma revisão integrativa, que busca reunir e resumir o conhecimento científico já produzido sobre o tema investigado, ou seja, permite buscar, avaliar e sintetizar as evidências disponíveis para contribuir com o desenvolvimento do conhecimento na temática ${ }^{8}$.

Para sua elaboração, faz-se necessário percorrer seis etapas distintas, similares às etapas decorridas na pesquisa convencional. A primeira etapa refere-se ao estabelecimento da questão norteadora que envolverá toda pesquisa, a escolha e definição do tema e a identificação dos descritores. A questão norteadora foi: "Quais as contribuições dos estudos científicos sobre Consulta de Enfermagem, com uso de habilidades empáticas, para clientes com doença cardiovascular em nível ambulatorial?". Utilizaram-se como descritores: "Assistência ambulatorial", cruzando-o com os descritores "Enfermagem Cardiovascular" e "Empatia".

A busca ocorreu em março de 2017, considerando-se a Biblioteca Virtual em Saúde (BVS). Num primeiro momento se utilizou os termos sem filtros e depois se aplicou ano de publicação entre 2012 e 2016, estarem nos idiomas: português, inglês e espanhol e estarem disponíveis os textos completos. Foram excluídas da análise monografias, teses e dissertações e as 
duplicações (mantendo-se apenas um quando as bases se repetiam.

Num terceiro momento de leitura de todos artigos, considerou-se apenas aqueles com relevância a temática de interesse. Para definição dos níveis de evidência dos artigos foi utilizado a seguinte hierarquização ${ }^{8}$ :

- Nível 1: evidências resultantes da metaanálise de múltiplos estudos clínicos controlados e randomizados;

- Nível 2: evidências obtidas em estudos individuais com delineamento experimental;

- Nível 3: evidências de estudos quaseexperimentais;

- Nível 4: evidências de estudos descritivos (não-experimentais) ou com abordagem qualitativa;
- Nível 5: evidências provenientes de relatos de caso ou de experiência;

- Nível 6: evidências baseadas em opiniões de especialistas.

Os artigos considerados foram colocados num quadro que considerou: ano, autores, local de publicação, local do estudo, nível de evidência e título e, após foram categorizados e, quando necessários subcategorizados.

\section{RESULTADOS}

Conforme o Quadro 1 encontrou-se 393 artigos na primeira busca.

Quadro 1. Artigos encontrados apenas pelos descritores na BVS. Rio de Janeiro, março de 2017.

\begin{tabular}{|l|c|}
\hline \multicolumn{1}{|c|}{ DESCRITORES } & TOTAL \\
\hline Assistência ambulatorial and Enfermagem cardiovascular & 226 \\
\hline Assistência ambulatorial and empatia & 146 \\
\hline Enfermagem Cardiovascular and Empatia & 21 \\
\hline Total & 393 \\
\hline
\end{tabular}

Ao se considerar ano de publicação disponíveis os textos completos e os fatores entre 2012 e 2016, estarem nos idiomas: português, inglês e espanhol e estarem de exclusão obteve-se 55 artigos.

Quadro 2 - Artigos encontrados na BVS que relacionam os descritores entre 2012 a 2016. Rio de Janeiro, março de 2017.

\begin{tabular}{|l|c|}
\hline \multicolumn{1}{|c|}{ DESCRITORES } & TOTAL \\
\hline Assistência ambulatorial and Enfermagem cardiovascular & 27 \\
\hline Assistência ambulatorial and empatia & 24 \\
\hline Enfermagem Cardiovascular and Empatia & 4 \\
\hline TOTAL & $\mathbf{5 5}$ \\
\hline
\end{tabular}

Ao se considerar as publicações disposição online atingiu-se 15 artigos analisadas quanto à relevância, duplicidade e conforme Figura 1.

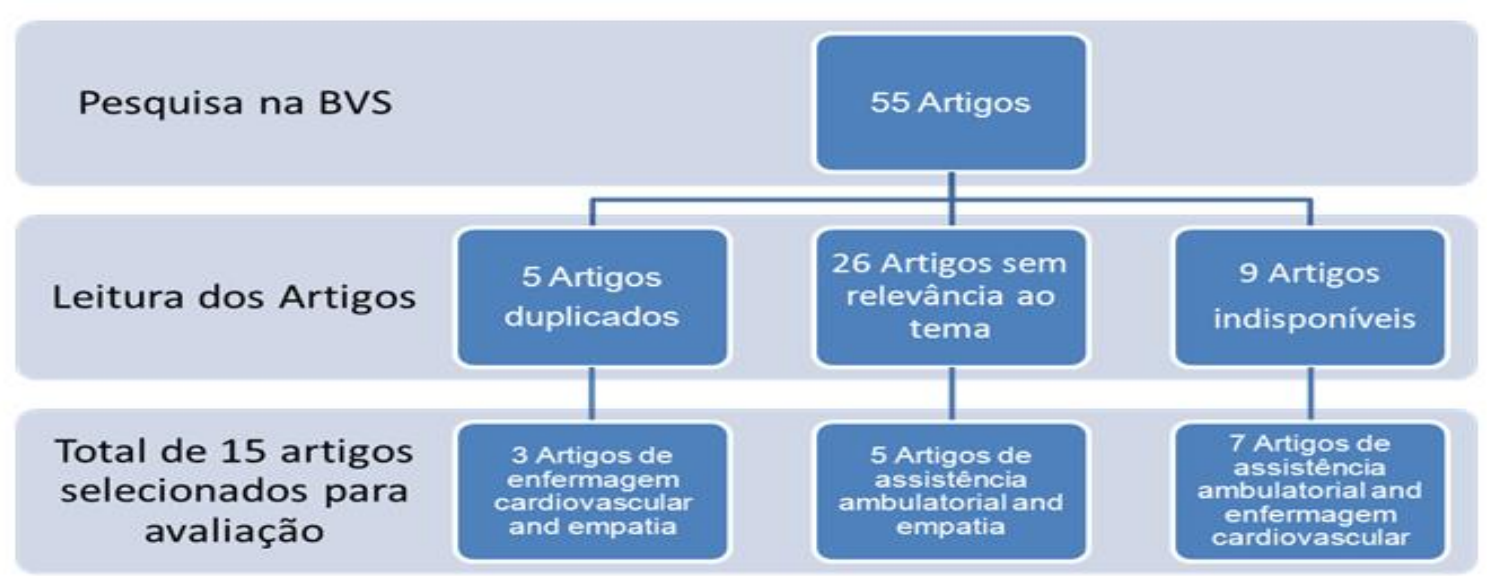

Figura 1. Artigos considerados na BVS conforme descritores - 2012 a 2016. Rio de Janeiro, março de 2017. 
Quadro 3. Artigos com a temática Empatia e Enfermagem Cardiovascular na Consulta de Enfermagem entre 2012 a 2016. Rio de Janeiro, março de 2017.

\begin{tabular}{|c|c|c|c|c|c|c|c|}
\hline 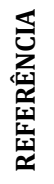 & $\underset{z}{z}$ & AUTORES & $\begin{array}{l}\text { LOCAL DE } \\
\text { PUBLICAÇÃOO }\end{array}$ & $\stackrel{n}{2}$ & MÉTODO & $\begin{array}{l}\text { NÍVEL DE } \\
\text { EVIDÊNCIA }\end{array}$ & TÍTULO \\
\hline 28 & 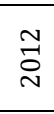 & Bala et al. & $\begin{array}{l}\text { Wiley Online } \\
\text { Library }\end{array}$ & 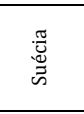 & $\begin{array}{c}\text { Qualitativo, } \\
\text { Descritivo e } \\
\text { Exploratório }\end{array}$ & Nível 4 & $\begin{array}{l}\text { The Experience of Care at Nurse- } \\
\text { Led Rheumatology Clinics }\end{array}$ \\
\hline 26 & $\stackrel{m}{\stackrel{n}{\sim}}$ & $\begin{array}{l}\text { Jorstad et } \\
\quad \text { al. }\end{array}$ & Heart & $\begin{array}{l}\frac{\pi}{0} \\
\stackrel{\pi}{0} \\
\frac{0}{0} \\
1\end{array}$ & $\begin{array}{c}\text { Quase- } \\
\text { Experimental }\end{array}$ & Nível 3 & $\begin{array}{l}\text { Effect of a nurse-coordinated } \\
\text { prevention programme on } \\
\text { cardiovascular risk after an acute } \\
\text { coronary syndrome: main results } \\
\text { of the RESPONSE randomised } \\
\text { trial }\end{array}$ \\
\hline 21 & $\stackrel{m}{\stackrel{n}{N}}$ & $\begin{array}{l}\text { Garcia et } \\
\quad \text { al. }\end{array}$ & $\begin{array}{c}\text { Fertility and } \\
\text { Sterility by } \\
\text { American Society } \\
\text { for Reproductive } \\
\text { Medicine } \\
\end{array}$ & 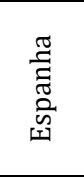 & $\begin{array}{c}\text { Quase - } \\
\text { Experimental }\end{array}$ & Nível 3 & $\begin{array}{l}\text { Training in empathic skills } \\
\text { improves the patient-physician } \\
\text { relationship during the first } \\
\text { consultation in a fertility clinic }\end{array}$ \\
\hline 11 & $\stackrel{m}{\stackrel{n}{\sim}}$ & $\begin{array}{l}\text { Macedo; } \\
\text { Sena; } \\
\text { Miranda }\end{array}$ & $\begin{array}{l}\text { Revista Brasileira } \\
\text { de Enfermagem }\end{array}$ & $\begin{array}{l}\overline{\vec{g}} \\
\overline{0} \\
\overline{0}\end{array}$ & $\begin{array}{l}\text { Qualitativo, } \\
\text { Descritivo e } \\
\text { Exploratório }\end{array}$ & Nível 4 & $\begin{array}{l}\text { Consulta de enfermagem ao } \\
\text { paciente com HIV: perspectivas e } \\
\text { desafios sob a ótica de } \\
\text { enfermeiros }\end{array}$ \\
\hline 19 & 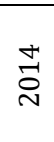 & $\begin{array}{l}\text { Hendriks } \\
\text { et al. }\end{array}$ & $\begin{array}{l}\text { Behalf of European } \\
\text { Society of } \\
\text { Cardiology }\end{array}$ & $\begin{array}{l}\frac{\pi}{0} \\
\frac{\pi}{\pi} \\
\frac{\pi}{0}\end{array}$ & $\begin{array}{c}\text { Quase- } \\
\text { Experimental }\end{array}$ & Nível 3 & $\begin{array}{l}\text { The effect of a nurse-led } \\
\text { integrated chronic care approach } \\
\text { on quality of life in patients with } \\
\text { atrial fibrillation }\end{array}$ \\
\hline 22 & 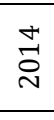 & $\begin{array}{l}\text { Oterhals } \\
\text { et al. }\end{array}$ & $\begin{array}{c}\text { European Journal of } \\
\text { Cardiovascular } \\
\text { Nursing }\end{array}$ & 怘 & $\begin{array}{l}\text { Quantitativo e } \\
\text { Descritivo }\end{array}$ & Nível 4 & $\begin{array}{l}\text { European Cardiac nurses' current } \\
\text { practice and knowledge on } \\
\text { anticoagulation therapy }\end{array}$ \\
\hline 24 & $\underset{\stackrel{D}{S}}{\stackrel{+}{S}}$ & $\begin{array}{l}\text { Larsen; } \\
\text { Neighbour }\end{array}$ & $\begin{array}{l}\text { British Journal of } \\
\text { General Practice }\end{array}$ & 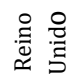 & $\begin{array}{l}\text { Análise de } \\
\text { Especialista }\end{array}$ & Nível 6 & $\begin{array}{l}\text { Five Cards: a simple guide to } \\
\text { beginning the consultation }\end{array}$ \\
\hline 13 & $\stackrel{+}{\stackrel{i}{\sim}}$ & $\begin{array}{l}\text { Amorim et } \\
\text { al. }\end{array}$ & $\begin{array}{l}\text { Revista Brasileira } \\
\text { de Enfermagem }\end{array}$ & $\begin{array}{l}\overline{\widetilde{n}} \\
\overline{\mathscr{n}} \\
\bar{m}\end{array}$ & $\begin{array}{l}\text { Qualitativo } \\
\text { delineado em } \\
\text { Estudo de } \\
\text { Caso }\end{array}$ & Nível 4 & $\begin{array}{l}\text { Cuidado sistematizado em pré- } \\
\text { operatório cardíaco: Teoria do } \\
\text { Cuidado Transpessoal na } \\
\text { perspectiva de enfermeiros e } \\
\text { usuários }\end{array}$ \\
\hline 23 & 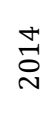 & $\begin{array}{l}\text { Albarran } \\
\text { et al. }\end{array}$ & $\begin{array}{l}\text { European Journal of } \\
\text { Cardiovascular } \\
\text { Nursing }\end{array}$ & : & $\begin{array}{l}\text { Qualitativo e } \\
\text { Descritivo }\end{array}$ & Nível 4 & $\begin{array}{l}\text { Patients' perspectives on the } \\
\text { educational preparation of } \\
\text { cardiac nurses }\end{array}$ \\
\hline 14 & 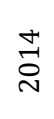 & $\begin{array}{l}\text { Komatsu; } \\
\text { Yagasaki }\end{array}$ & $\begin{array}{l}\text { European Journal of } \\
\text { Oncology Nursing }\end{array}$ & $\begin{array}{l}\frac{0}{2} \\
\underset{\pi}{\pi} \\
\stackrel{\pi}{0}\end{array}$ & Qualitativo & Nível 4 & $\begin{array}{l}\text { The Power of nursing: Guiding } \\
\text { patients through a journey of } \\
\text { uncertainty }\end{array}$ \\
\hline 12 & $\stackrel{\operatorname{Ln}}{\stackrel{2}{N}}$ & $\begin{array}{l}\text { Andersso } \\
\mathrm{n} \text { et al. }\end{array}$ & $\begin{array}{l}\text { Journal of Clinical } \\
\text { Nursing }\end{array}$ & 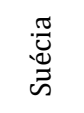 & $\begin{array}{l}\text { Qualitativo e } \\
\text { Descritivo }\end{array}$ & Nível 4 & $\begin{array}{l}\text { Registered nurses views of caring } \\
\text { in coronary care - a deductive and } \\
\text { inductive content analysis }\end{array}$ \\
\hline 27 & $\stackrel{\text { nn }}{\stackrel{2}{N}}$ & $\begin{array}{c}\text { Smith et } \\
\text { al. }\end{array}$ & $\begin{array}{l}\text { Journal } \\
\text { Cardiovascular } \\
\text { Nursing }\end{array}$ & 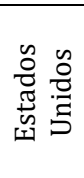 & $\begin{array}{c}\text { Quase- } \\
\text { Experimental }\end{array}$ & Nível 3 & $\begin{array}{l}\text { Nurse-led Multidisciplinary Heart } \\
\text { Failure Group Clinic } \\
\text { Appointments: Methods, } \\
\text { Materials and Outcomes Used in } \\
\text { the Clinical Trial }\end{array}$ \\
\hline 20 & $\stackrel{n}{\stackrel{2}{2}}$ & $\begin{array}{l}\text { Cooper; } \\
\text { Zimmerm } \\
\text { an }\end{array}$ & $\begin{array}{l}\text { Public Health } \\
\text { Nursing }\end{array}$ & 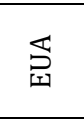 & $\begin{array}{c}\text { Quase- } \\
\text { Experimental }\end{array}$ & Nível 3 & $\begin{array}{l}\text { The Evaluation of a Regional Faith } \\
\text { Community Network's Million } \\
\text { Hearts Program }\end{array}$ \\
\hline 31 & 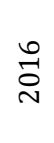 & $\begin{array}{l}\text { Bessa; } \\
\text { Mesquita; } \\
\text { Stipp }\end{array}$ & $\begin{array}{l}\text { Revista Online de } \\
\text { Pesquisa Cuidado é } \\
\text { Fundamental }\end{array}$ & 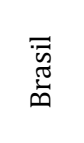 & $\begin{array}{l}\text { Quantitativo, } \\
\text { Descritivo e } \\
\text { Exploratório }\end{array}$ & Nível 4 & $\begin{array}{l}\text { Saúde do homem e doença } \\
\text { cardiovascular: gerenciamento do } \\
\text { cuidado de enfermagem em nível } \\
\text { ambulatorial }\end{array}$ \\
\hline 29 & 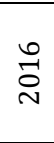 & $\begin{array}{l}\text { Ferguson } \\
\text { et al. }\end{array}$ & $\begin{array}{l}\text { BMC Medical } \\
\text { Education }\end{array}$ & 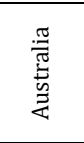 & $\begin{array}{l}\text { Quantitativo e } \\
\text { Descritivo }\end{array}$ & Nível 4 & $\begin{array}{l}\text { Education and practice gaps on } \\
\text { atrial fibrillation and } \\
\text { anticoagulation: a survey of } \\
\text { cardiovascular nurses }\end{array}$ \\
\hline
\end{tabular}


Dos artigos selecionados, apenas 3 foram elaborados no Brasil, demonstrando que a temática ainda precisa ser melhor explorada no país. Também cabe ressaltar que apenas 5 artigos são do tipo quaseexperimentais, sendo a maioria com baixo nível de evidência do tipo descritivo, nãointervencionista.

A partir da leitura destes artigos, as publicações foram classificadas em duas categorias:

- A Consulta de Enfermagem; e,

- A avaliação dos clientes sobre as práticas do enfermeiro na Consulta de Enfermagem.

\section{DISCUSSÃO}

\section{Categoria 1: A Consulta de Enfermagem}

A consulta de enfermagem é uma atividade privativa do enfermeiro e está prevista na Lei do Exercício Profissional de Enfermagem, Lei $\mathrm{N}^{\circ} 7498$ de 25 de Junho de $1986^{9}$.

A Resolução do COFEN (Conselho Federal de Enfermagem) no 358, de 15 de outubro de 2009 define que o Processo de Enfermagem quando realizado em instituições prestadoras de serviços ambulatoriais de saúde corresponde a Consulta de Enfermagem, que deve ser baseada em um suporte teórico, de modo sistemático e possui 5 etapas interligadas ${ }^{10}$ :

- Histórico de enfermagem - processo deliberado, sistemático e contínuo, realizado com o auxílio de métodos e técnicas variadas, que tem por finalidade a obtenção de informações sobre a pessoa, família ou coletividade humana e sobre suas respostas em um dado momento do processo saúde e doença;

- Diagnóstico de enfermagem - processo de interpretação e agrupamento dos dados coletados na primeira etapa, que culmina com a tomada de decisão sobre os conceitos diagnósticos de enfermagem que representam, com mais exatidão, as respostas da pessoa, família ou coletividade humana em um dado momento do processo saúde e doença; e que constituem a base para a seleção das ações ou intervenções com as quais se objetiva alcançar os resultados esperados;
- Planejamento de enfermagem determinação dos resultados que se espera alcançar; e das ações ou intervenções de enfermagem que serão realizadas face às respostas da pessoa, família ou coletividade humana em um dado momento do processo saúde e doença, identificadas na etapa de Diagnóstico de Enfermagem;

- Implementação - realização das ações ou intervenções determinadas na etapa de Planejamento de Enfermagem;

- Avaliação de enfermagem - processo deliberado, sistemático e contínuo de verificação de mudanças nas respostas da pessoa, família ou coletividade humana em um dado momento do processo saúde doença, para determinar se as ações ou intervenções de enfermagem alcançaram o resultado esperado; e de verificação da necessidade de mudanças ou adaptações nas etapas do Processo de Enfermagem.

A consulta de enfermagem é utilizada para propagar informações e realizar orientações sobre a patologia e para convocar o cliente para participação ativa do cuidado. Além disso, através dela, torna-se possível promover o apoio, acolhimento, a escuta e o diálogo com o paciente, sendo um momento educativo importante para troca de saberes e estreitamento do vínculo11.

Para melhor discussão dos achados, esta categoria foi dividida em duas subcategorias: A prática da consulta de enfermagem quanto aos aspectos subjetivos do cuidar e a Práxis da consulta de enfermagem: a experiência como ela é ou como deveria ser.

\section{Subcategoria 1.1: A prática da consulta de enfermagem quanto aos aspectos subjetivos do cuidar}

A consulta de enfermagem deve ultrapassar os limites da informação e orientação ao cliente, proporcionando real momento de transformação do indivíduo, permitindo que este se sinta acolhido, compreendido e à vontade para dialogar sobre dúvidas, inquietações e angústias ${ }^{11}$.

0 cuidado de enfermagem precisa ocorrer através do diálogo e cooperação com o paciente, sendo um importante facilitador prestar atenção nos pensamentos e desejos do 
cliente. Além disso, acrescenta-se a necessidade de se desenvolver intervenções de acordo com necessidades e recursos do indivíduo e sua vida cotidiana ${ }^{12}$.

Como parte do trabalho do enfermeiro está a compreensão do seu cliente. Uma pesquisa demonstrou que a escuta foi principal mecanismo utilizado durante a Consulta de Enfermagem para construir uma relação de empatia e confiança com o cliente, fundamental para que seja oferecido apoio emocional e o tratamento se estabeleça com eficácia ${ }^{11}$. Além disto, que a escuta sensível e o diálogo aberto possibilitam colocar o enfermeiro em um papel diferenciado, sendo percebido como uma pessoa mais próxima, alguém em que se pode confiar, muito além de ser somente um profissional de saúde ${ }^{11}$.

A primeira parte de qualquer atendimento de enfermagem, inclusive na consulta deve ser ouvir o cliente, instrumento necessário para conhecer adequadamente uma pessoa. Este ouvir se une ao ser de quem se cuida, em uma relação que percebe o todo formado pela mente, corpo e espírito. Perceber as necessidades físicas, emocionais, espirituais e sociais do cliente, demanda do profissional uma constante atualização de subsídios teóricos capazes de oferecer resultados favoráveis a partir dos diagnósticos e intervenções ${ }^{13}$.

Um estudo realizado no Japão descreveu que os enfermeiros fizeram esforços substanciais para se conectar com seus clientes, para entendê-los. Tais esforços começaram pela adoção de uma atitude "Nós nos preocupamos com você" para mostrar acolhimento aos seus clientes. Ademais não só ouviam seus clientes, mas também faziam uma avaliação para descobrir e compartilhar suas necessidades, considerando seus valores e desejos. Além disso, insistiram que escuta atenta não seria suficiente para ajudar seus clientes, mas apenas o começo ${ }^{14}$.

Habilidades empáticas como compaixão, escuta ativa e acolhedora, e a compreensão dos agravos à saúde, para além de sinais e sintomas são fundamentais para a construção de vínculo de confiança, essencial na construção e manutenção do vínculo terapêutico ${ }^{15}$.
A empatia é o resultado de uma construção multidimensional que envolve componentes afetivos (capacidade de se sensibilizar diante da situação vivida pelo outro que pode ser compartilhada ou não), cognitivos (capacidade de se fazer uma leitura da perspectiva do outro. 0 que ele está pensando/sentindo?) e comportamentais (capacidade de comunicar de maneira verbal e não verbal o entendimento da situação) ${ }^{15}$.

Em relação ao aspecto cognitivo, foi realizado um estudo ${ }^{16}$ com estudantes de medicina, onde se relacionou a Escala Jefferson de empatia médica e o Reconhecimento de Expressões Faciais de emoções básicas (REF). Como resultado se encontrou que pessoas com maior empatia reconheceram melhor a expressão básica da tristeza, demostrando que uma pessoa empática consegue identificar melhor 0 sofrimento do outro.

A empatia é a capacidade de entender aquilo que uma pessoa está sentindo e transmitir-lhe compreensão, mantendo ao mesmo tempo certa objetividade para poder prestar a ajuda necessária ${ }^{17}$. Pois, para compreender o sentimento de um indivíduo e realizar intervenções de acordo com as necessidades e recursos da pessoa, são necessários uma escuta sensível e diálogo. Além disso, intervindo com objetividade e de maneira correta, o cliente confiará no profissional, por ter sido compreendido e também porque presenciará uma melhora na sua qualidade de vida.

É importante desenvolver um plano de cuidados que não se restrinja às condutas técnico-científicas, necessários ao controle da patologia e à modelagem de comportamentos, mas que consiga contemplar as subjetividades imersas no processo de adoecimento e que se constituem em situações propulsoras de angústia, medo e sofrimento ${ }^{11}$.

A Organização Mundial da Saúde definiu a saúde como sendo o estado de completo bem-estar físico, mental e social e não somente ausência de afecções e enfermidades ${ }^{18}$. Sendo assim, torna-se imprescindível pesquisar todas as necessidades do cliente, inclusive suas necessidades psicológicas, pois se entende 
que o conceito de saúde transcende a ausência de doença. A consulta de enfermagem, por sua vez, deve contemplar essas questões, visando o cuidado integral com manutenção da qualidade de vida e seu bem-estar mental.

Relacionado ao bem-estar mental, em estudo com clientes com fibrilação atrial, foi demonstrado que fatores psicológicos como angústia e depressão aumentam o risco de desenvolvimento da doença, assim como de outras doenças cardiovasculares, além de diminuir a qualidade de vida do indivíduo, mostrando que o cuidado integral protagonizado pelo enfermeiro possui um papel vital. Demonstrou-se ainda que um menor conhecimento do cliente sobre a patologia pode diminuir sua qualidade de vida por aumentar a chance de angústia $e$ depressão ${ }^{19}$.

As orientações do enfermeiro são importantes, pois sanam dúvidas do cliente sobre sua patologia, medicamentos, tratamentos, e outras questões, pois é comum ao ser humano o medo do desconhecido.

Ainda sobre as questões psicológicas, um estudo mostrou que seria interessante olhar as práticas espirituais durante o cuidado de enfermagem para auxiliar na gestão do estresse $^{20}$.

Uma pesquisa ${ }^{21}$ a partir de uma atividade de treinamento para profissionais, com os seguintes tópicos: empatia como um todo, inteligência emocional, comunicação verbal e não-verbal, escuta ativa e tipos de comportamento resultou numa maior satisfação dos pacientes nos quesitos informação de qualidade, habilidades de comunicação, tempo dedicado e experiência.

$\mathrm{Na}$ leitura dos artigos, verificou-se que a prática objetiva dos cuidados de enfermagem deve ser posterior a criação de vínculo com o cliente atendido. Faz-se necessário ganhar sua confiança e atender suas demandas além da patologia, para tornálo participante ativo do cuidado. Para atingir este objetivo é necessário o desenvolvimento de habilidades empáticas por parte dos enfermeiros.
Subcategoria 1.2: Práxis da consulta de enfermagem: a experiência como ela é ou como deveria ser

Para implementar intervenções e realizar orientações, se faz necessário um espaço físico e tempo adequados, além de continuidade. Condições de trabalho deficitárias podem tornar 0 cuidado com 0 paciente marginalizado ${ }^{12}$.

Os enfermeiros têm um papel fundamental para orientar os clientes sobre o potencial e os efeitos colaterais dos medicamentos e a importância de aderir aos tratamentos ${ }^{16}$. Em estudo com pessoas em uso de anticoagulante, a educação para administração correta do fármaco e seus efeitos secundários foi a atribuição mais comum para os enfermeiros ${ }^{22}$.

Além das orientações sobre os fármacos, outras questões primordiais a serem abordados pelos enfermeiros durante a consulta incluem: fatores de risco para doença cardíaca, dieta, exercício físico, saúde sexual, estresse e mudanças de estilo de vida ${ }^{12}$. Para realizar tais orientações, é fundamental o profissional possuir uma compreensão abrangente das condições cardiovasculares, além de conhecimento para implementar medida efetivas ${ }^{23}$.

Para realizar a consulta, o enfermeiro precisa utilizar diversas ferramentas, com vista a manter um acompanhamento contínuo, individual e eficaz.

A Sistematização da Assistência de Enfermagem (SAE), que enquanto processo organizacional é capaz de oferecer subsídios para o desenvolvimento de métodos interdisciplinares e humanizados de cuidado, representa importante conquista no campo assistencial da enfermagem. Porém, o profissional imbuído nesse processo deve considerar que a seleção de intervenções deve ser dirigida ao sujeito que possui o diagnóstico (não ao diagnóstico) e influenciada pelas condições do cliente, disponibilidade de recursos, custos e experiência da equipe ${ }^{11}$.

Reforça-se que a SAE orientada por um referencial teórico e pelas taxonomias confere clareza ao processo de trabalho do enfermeiro. A satisfação do usuário pode ser 
verificada pelo profissional a partir da avaliação dos resultados (NOC) das intervenções (NIC) propostas, revelando também a efetividade ou não das medidas ${ }^{13}$.

Além da SAE, outros recursos podem e precisam ser utilizados para garantir um efeito positivo da consulta de enfermagem. Em pesquisa realizada no Reino Unido ${ }^{24}$, foram descritas estratégias a serem utilizadas durante uma consulta médica, que podem também ser utilizadas pelos enfermeiros. A consulta deve ser realizada em 3 partes, na qual a primeira deve ser parte do paciente (com liberdade para expressar suas experiências anteriores a consulta, pensamentos, ideias, preocupações e expectativas), a segunda a parte do profissional (realização da investigação e transformação da narrativa leiga em linguagem técnica) e a terceira a parte compartilhada (para definição do plano de ação) ${ }^{24}$.

0 mesmo estudo ${ }^{24}$ considerou que para obter as ideias, preocupações e expectativas dos clientes, deveriam se detalhar perguntas encaminhadoras como: "O que você acha que está causando isso?" (ideias); "Tem alguma coisa em particular preocupando você?" (preocupações); "Como você gostaria que eu ajudasse?" (expectativa). Além disso, indicaram frases a serem ditas para incentivar o paciente a falar: "Obrigado por me falar isso"; "Fale mais"; "Isso é útil"; "Fico feliz que tenha mencionado isso"; "Consigo ver que isso te preocupa bastante"24. Tais estratégias podem auxiliar o profissional a conhecer ainda mais seu cliente e propor intervenções específicas para cada indivíduo.

Em sua Teoria de Enfermagem, Travelbee segmentou em fases distintas o processo de relacionamento enfermeiropaciente, com algumas características da divisão realizada por Larsen e Neighbour para consulta, tendo como principal diferença a parte compartilhada entre o cliente e o profissional que tem como foco o autocuidado e o cuidado de si ${ }^{25}$. Dividiu em 4 fases, sendo: 1a - Fase de pré-interação: É a fase em que o enfermeiro identifica a pessoa e obtém as informações necessárias para o desenvolvimento do relacionamento (reconhece seus sentimentos, pensamentos e comportamentos);

2 - Fase introdutória ou de orientação: É o momento de conhecer e compreender o outro, sua singularidade; é um momento único em que ambos experienciam crescimento pessoal;

$3^{\text {a }}$ - Fase de identidades emergentes: caracteriza-se pela resolução dos problemas encontrados na fase anterior, ou seja, é o desenvolvimento pleno do relacionamento pessoa-a-pessoa;

$4^{\text {a }}$ - Fase término: uma das suas características importantes é que enfermeiro deve lembrar o paciente do compromisso que fizeram; analisar o alcance dos objetivos propostos, a importância e motivos do término e prepará-lo para continuar independente no que for possível ${ }^{25}$.

Reforçando a importância da consulta de enfermagem, estudos demonstram que pessoas com doenças cardiovasculares atendidas continuamente por enfermeiros apresentam um menor número de reinternações por complicações relativas à sua doença de base $19,26,27$, causando um benefício individual e também coletivo (devido ao alto custo de internações, licenças médicas e perda de produtividade).

Em pesquisa realizada na Holanda, um dos motivos para a diminuição das internações foi o aumento de $37 \%$ na proporção dos pacientes que foram classificados como tendo bom controle dos fatores de risco, avaliado por duas escalas diferentes $^{9}$. Outro esudo ${ }^{12}$ ressaltou a elevação do conhecimento sobre a patologia como primordial, melhorando ainda a qualidade de vida de seus pesquisados. Destaca-se também a melhora do autocuidado e maior aderência ao tratamento ${ }^{13}$.

Para seu trabalho ser efetivo, enfermeiros precisam ter conhecimento para responder a perguntas básicas e fornecer boas orientações ${ }^{22,28}$. Parece óbvio, mas um estudo na Nova Zelândia e Austrália demonstrou que enfermeiros cardiovasculares possuíam conhecimento insuficiente sobre terapia anticoagulante oral, na interação da warfarina com outros medicamentos. A falta de conhecimento sobre esses tópicos pode 
contribuir para o aconselhamento e educação inadequados. Além disso, a comunicação de informações imprecisa pode reforçar mitos e equívocos em torno da anticoagulação ${ }^{29}$.

Para melhorar tal fato, a pesquisa propõe que todos os enfermeiros devem participar em pelo menos 20 horas de desenvolvimento profissional contínuo por ano sobre anticoagulação, incluindo risco de sangramento e modificação do estilo de vida que podem ajudar na manutenção de um bom padrão da assistência e o desenvolvimento da enfermagem cardiovascular ${ }^{29}$.

Não existe nenhuma resolução do COFEN que indique a carga horária mínima que precisa ser disponibilizada para atualização dos profissionais de enfermagem. A Resolução COFEN 293/2004 apenas indica que o responsável técnico pela equipe enfermagem deve ter de 3 a $5 \%$ do quadro geral da equipe de enfermagem para cobertura de situações relacionadas à rotatividade de pessoal e participação de programas de educação continuada. Fato que precisa ser visto, pois é imprescindível manter os profissionais atualizados para que o cuidado de enfermagem seja feito de maneira correta, precisa, segura e sem erros.

A Consulta de Enfermagem precisa ser realizada num local adequado, de forma sistematizada, fundamentada por uma teoria, com a utilização de uma taxonomia para dar clareza ao processo de enfermagem. 0 Enfermeiro necessita estar preparado para dar orientações sobre a patologia; fatores de risco; mudanças no estilo de vida; uso dos fármacos, seus efeitos colaterais e interações. Para tal, deve dividir a consulta de maneira que o cliente consiga expressar todas as suas dúvidas, pensamentos e angústias e criar estratégias em conjunto de acordo com cada indivíduo.

\section{Categoria 2: A avaliação dos clientes sobre a prática do enfermeiro na Consulta de Enfermagem}

Em pesquisa realizada no Reino Unido os pacientes informaram que desejavam ser cuidados por pessoas que eram tecnicamente competentes e experientes, mas, de igual importância, eles queriam enfermeiros quem poderiam também demonstrar sua compaixão e responder às preocupações emocionais através de efetivas habilidades interpessoais ${ }^{23}$.

Disseram ainda que consideraram que os enfermeiros cardíacos devem ser equipados e preparados para apoiar, guiar e engajar os pacientes nas várias fases de reabilitação. Isso inclui habilidades interpessoais, modelos de mudança de comportamento, habilidades para aconselhamento e dotação de estratégias para promover um nível adequado de otimismo entre os pacientes sobre o futuro ${ }^{23}$.

Tais afirmações mostram a importância do cuidado objetivo da patologia, mas que a parte subjetiva é também de extrema relevância, inclusive para garantir que as orientações sejam bem entendidas e seguidas. Fica claro que os clientes querem ser vistos em seu todo, não apenas a sua doença.

$\mathrm{Na}$ mesma pesquisa, pacientes expressaram uma preocupação especial relacionada com compreensão, pelos enfermeiros, da farmacologia e como a informação sobre seus medicamentos foi transmitida. Os participantes sugeriram que quando eles tinham perguntas sobre os seus medicamentos, os enfermeiros eram frequentemente incapazes de explicar os efeitos da droga e efeitos colaterais associados $^{23}$.

Já em pesquisa realizada na Suécia ${ }^{28}$, foi relatado que o cuidado focado na educação do paciente foi descrito como competente porque os enfermeiros eram professores proficientes tanto na parte teórica como na prática. 0 cuidado foi experimentado como profissional e motivador quando os enfermeiros ofereceram uma combinação de informação oral e escrita, verbalizaram benefícios e discutiram vantagens e desvantagens, tornando-se mais fácil de seguir a orientação.

Estas pesquisas mostram a necessidade de atualização contínua por parte dos profissionais, para garantir que os clientes criem confiança, tão importante para geração do vínculo necessário para um atendimento de qualidade.

Em estudo realizado com enfermeiros brasileiros $^{30}$ foi realçado por eles a 
importância e a necessidade do conhecimento técnico-científico. Todavia, ressaltaram a relevância de incluir nas ações educativas, aspectos que contemplam a mudança de paradigma na formação profissional e na atenção à saúde. Os participantes destacaram a valorização profissional e a autonomia para a realização das atividades relacionadas à competência do enfermeiro, ressaltando a educação permanente como estratégia para aquisição e atualização de conhecimentos e formação de agentes multiplicadores junto aos usuários e à equipe de saúde.

Os enfermeiros já compreenderam a necessidade de olhar o cliente para além de sua patologia, querendo adquirir conhecimentos, não apenas técnico-científico, mas também de relação interpessoal. Ademais se mostram dispostos, por meio da educação permanente, a obter maior instrução para prestar uma assistência de qualidade ${ }^{30}$.

A utilização de habilidades empáticas foi apreciada pelos clientes participantes em três pesquisas, avaliando como bons enfermeiros aqueles profissionais que os atenderam com empatia, relacionando esta ao fato de que os enfermeiros levavam as necessidades dos participantes a sério ${ }^{21,22,28}$.

Estudo sobre o cuidado em uma clínica de reumatologia, conduzido por enfermeiros, relatou que os participantes expressaram que foram afetados pela forma como foram recebidos, pelo que foi mostrado e o que foi dito. As expressões faciais e linguagem corporal dos enfermeiros tiveram um impacto sobre esta experiência e também a forma como eles se comunicavam. Por meio do comportamento focado nos indivíduos, os participantes foram reconhecidos, criando condições para um bom relacionamento e estabelecendo uma atmosfera calma, acolhedora e amigável. 0 ambiente físico também foi descrito como importante ${ }^{28}$.

Em pesquisa com clientes com Insuficiência Cardíaca (IC), a maioria dos participantes escreveu comentários, indicando que "falar sobre" e "compartilhar opiniões com os outros" eram as melhores maneiras de aprender a lidar e gerenciar sua IC ${ }^{27}$, o que mostra a importância do diálogo para manejo da doença.
No tocante aos métodos preventivos para doenças cardiovasculares se observou que os serviços de saúde são a maior fonte de informação para os clientes. Os usuários enxergam o enfermeiro como um profissional acolhedor, possibilitando maior aproximação durante a realização do cuidado, o que favorece a estabelecer uma comunicação satisfatória e promover a educação em saúde ${ }^{31}$. Tal achado demonstra a importância do enfermeiro na prevenção de DCV e controle de agravos.

Assim como as grandes empresas realizam pesquisas para avaliar a satisfação dos clientes com seus atendimentos e buscar suas reais necessidades, é necessário que o enfermeiro faça o mesmo para conseguir a efetividade da assistência prestada, além de se manter sempre atualizado para prestar orientações corretas aos usuários.

Soma-se o fato de as pessoas quererem se sentir importantes para o enfermeiro; desejam ser tratadas de forma empática, sendo ouvidas e compreendidas, criando uma relação maior que apenas de clienteprofissional.

\section{CONCLUSÃO}

Destaca-se nesta revisão a necessidade de o enfermeiro atuar pensando no sujeito em todos os seus aspectos (físico, psicológico, emocional, religioso, e outros.) e não só na patologia. Para isso, precisam possuir e/ou desenvolver habilidades empáticas que lhes possibilitem criar um vínculo com o cliente e uma relação de confiança.

Para fazer com que o cliente confie em seu trabalho e tenha efetividade nas intervenções, além de utilizar da empatia, o profissional precisar possuir o conhecimento técnico-científico necessário para atender todas as demandas possíveis do indivíduo. Para tal, precisa se atualizar constantemente, por meio da educação permanente e individualmente.

Mostrando a efetividade da assistência de enfermagem em nível ambulatorial, os estudos demonstraram um menor número de internações em clientes acompanhados continuamente por enfermeiros, trazendo um 
bem para o indivíduo, para sua família e para coletividade.

Sobre as limitações dessa revisão, o período temporal considerado foi de 2012 a 2016 e neste intervalo foi encontrado um número reduzido de artigos na base de dados escolhida, apesar da inclusão de artigos escritos em português, inglês e espanhol.

Assim, novas pesquisas contemplando distintas bases de dados e aumentando o período temporal podem contribuir para se delinear um cenário mais completo que o encontrado neste estudo. Além disso, torna-se necessário o desenvolvimento de mais pesquisas sobre a utilização da empatia no atendimento ambulatorial para clientes com doença cardiovascular, principalmente pesquisas que possuam maior nível de evidência, pois, nesta revisão, foram encontrados apenas 5 estudos quaseexperimentais.

\section{REFERÊNCIAS}

1. Andrade JS, Vieira MJ. Prática assistencial de enfermagem: problemas, perspectivas $\mathrm{e}$ necessidade de sistematização. Rev Bras Enferm. [Internet]. 2005[citado em 20 out 2017]; 58 (3):261-5. Disponível em: http://www.scielo.br/pdf/reben/v58n3/a02 v58n3.pdf

DOI: $\quad$ http://dx.doi.org/10.1590/S003471672005000300002

2. Ministério da Saúde (Br). Diretrizes para o cuidado das pessoas com doenças crônicas nas redes de atenção à saúde e nas linhas de cuidado prioritárias. Brasília, DF: Ministério da Saúde; 2013.

3. Araújo-Girão AL, Martins OGY, Gomes EB, Parente-Arruda L, Aires de Freitas CH. A interação no ensino clínico de enfermagem: reflexos no cuidado à pessoa com hipertensão arterial. Rev Salud Pública [Internet]. 2015 [citado em 20 out 2017]; 17(1): 47-60. Disponível em: http://www.scielo.org.co/pdf/rsap/v17n1/v 17n1a05.pdf

DOI: http://dx.doi.org/10.15446/rsap.v17n 1.47789

4. Conselho Federal de Enfermagem (Br). Resolução n. 311 de 08 de fevereiro de 2007. Aprova a Reformulação do Código de Ética dos
Profissionais de Enfermagem [Internet]. Brasília, DF: COFEN; 2007 [citado em 20 out 2017]. Disponível em: http://www.cofen.gov.br/resoluo-cofen3112007_4345.html

5. Mansur AP, Favarato D. Tendências da Taxa de mortalidade por doenças cardiovasculares no Brasil, 1980-2012. Arq Bras Cardiol. [Internet]. 2016 [citado em 25 out 2017]; 107(1):20-5. Disponível em: http://www.scielo.br/pdf/abc/2016nahead/ pt_0066-782X-abc-20160077.pdf

6. Instituto Brasileiro de Geografia e Estatísticas. Pesquisa Nacional de Saúde 2013: percepção do estado de saúde, estilos de vida e doenças crônicas. Rio de Janeiro: IBGE; 2014. 180p.

7. Siqueira ASE, Siqueira-Filho AG, Land MGP. Análise do impacto econômico das doenças cardiovasculares nos últimos cinco anos no Brasil. Arq Bras Cardiol. [Internet]. 2017 [citado 25 out 2017]; 109(1):39-46. Disponível em: http://www.scielo.br/pdf/abc/v109n1/pt_0 066-782X-abc-20170068.pdf DOI: http://dx.doi.org/10.5935/abc.20170068

8. Mendes KDS, Silveira RCCP, Galvão CM. Revisão integrativa: método de pesquisa para a incorporação de evidências na saúde e na enfermagem. Texto Contexto \& Enferm. [Internet]. 2008 [citado 25 out 2017]; 17(4):758-64. Disponível em: http://www.scielo.br/pdf/tce/v17n4/18.pdf DOI: $\quad$ http://dx.doi.org/10.1590/S010407072008000400018

9. Conselho Federal de Enfermagem (Br). Lei n. 7498 de 25 de junho de 1986. Dispõe sobre a regulamentação do exercício de enfermagem, e dá outras providências [Internet]. Brasília, DF: COFEN; 1986 [citado em 20 out 2017]. Disponível em: http://www.cofen.gov.br/lei-n-749886-de25-de-junho-de-1986_4161.html

10. Conselho Federal de Enfermagem (Br). Resolução n. 358 de 15 de outubro de 2009. Dispõe sobre a Sistematização da Assistência de Enfermagem e a implementação do Processo de Enfermagem em ambientes, públicos ou privados, em que ocorre o cuidado profissional de Enfermagem, e dá outras providências [Internet]. Brasília, DF: COFEN; 
2009 [citado em 20 out 2017]. Disponível em: http://www.cofen.gov.br/resoluo-cofen3582009_4384.html

11. Macêdo SM, Sena MCS, Miranda KCL. Consulta de enfermagem ao paciente com HIV: perspectivas e desafios sob a ótica de enfermeiros. Rev Bras Enferm. [Internet]. 2013 [citado em 26 out 2017]; 66(2):196-201. Disponível

em:

http://www.scielo.br/pdf/reben/v66n2/07. pdf DOI: http://dx.doi.org/10.1590/S003471672013000200007

12. Andersson EK, Sjöström-Strand A, Willman A, Borglin G. Registered nurses views of caring in coronary care-a deductive and inductive content analysis. J Clin Nurs. 2015; 24(23-24):3481-93.

13. Amorim TV, Arreguy-Sena C, Alves MS, Salimena AMO. Cuidado sistematizado em pré-operatório cardíaco: teoria do cuidado transpessoal na perspectiva de enfermeiros e usuários. Rev Bras Enferm. [Internet]. 2014 [citado em 27 out 2017]; 67(4):568-74. Disponível

em:

http://www.scielo.br/pdf/reben/v67n4/003 4-7167-reben-67-04-0568.pdf

DOI: http://dx.doi.org/10.1590/00347167.2014670411

14. Komatsu K, Yagasaki K. The power of nursing: guiding patients through a journey of uncertainty. Eur J Oncol Nurs. 2014; 18(4):419-24.

15. Kestenberg CCF. A habilidade empática é socialmente aprendida: um estudo experimental com graduando de enfermagem. Rev Enferm UERJ. 2013; 21(4):427-33.

16. Ávila RF, Morais D, Bomfim AJ, Chagas MHN. Empatia e reconhecimento de expressões faciais de emoções básicas e complexas em estudantes de medicina. J Bras Psiquiatr. [Internet]. 2016 [citado em 27 out 2017]; 65(3):209-14. Disponível em: http://www.scielo.br/pdf/jbpsiq/v65n3/00 47-2085-jbpsiq-65-3-0209.pdf

DOI: $\quad$ http://dx.doi.org/10.1590/00472085000000126

17. Fish S, Shelly JA. Cuidado espiritual do paciente. São Paulo: Umhe;1986. 190p.

18. World Health Organization. Global status report on non communicable diseases 2010 .
Geneva: World Health Organization; 2011. $176 \mathrm{p}$.

19. Hendriks JM, Wit RCHJ, Vrijhoef HJ, Prins MH, Pisters R, Tieleman RG. Nurse-led care vs usual care for patients with atrial fibrillation: results of a randomized trial of integrated chronic care vs routine clinical care in ambulatory patients with atrial fibrillation. Eur Heart J. 2012; 33(21):2692-9. 20. Cooper J, Zimmerman W. The evaluation of a regional faith community network's million hearts program. Public Health Nurs. 2016; 33(1):53-64.

21. García D, Bautista 0, Venereo L, Coll O, Vassena R, Vernaeve V. Training in empathic skills improves the patient-physician relationship during the first consultation in a fertility clinic. Fertil Steril. 2013; 99(5):14138.e1.

22. Oterhals K, Deaton C, De Geest S, Jaarsma $\mathrm{T}$, Lenzen M, Moons $\mathrm{P}$, et al. European cardiac nurses' current practice and knowledge on anticoagulation therapy. Eur J Cardiovasc Nurs. 2014; 13(3):261-9.

23. Albarran JW, Jones I, Lockyer L, Manns S, Cox H, Thompson DR. Patients' perspectives on the educational preparation of cardiac nurses. Eur J Cardiovasc Nurs. [Internet]. 2014 [citado em 27 out 2017]; 13(5):451-8. Disponível em: https://journals.sagepub.com/doi/pdf/10.11 77/1474515113507166

DOI: http://dx.doi.org/10.1177/1474515113507 166

24. Larsen JH, Neighbour R. Five cards: a simple guide to beginning the consultation. $\mathrm{Br}$ J Gen Pract. 2014; 64(620):150-1.

25. Torney AM, Alligood MR. Teóricas de enfermagem e sua obra (modelos e teorias de enfermagem). 5ed. Loures, PT: Lusociência Editora; 2004. 766p.

26. Jorstad HT, Von Birgelen C, Alings AM, Liem A, Van Dantzig JM, Jaarsma W, et al. Effect of a nurse-coordinated prevention programme on Joint ESC Guidelines 2381j cardiovascular risk after an acute coronary syndrome: main results of the RESPONSE randomised trial. Heart. 2013; 99(19):142130.

27. Smith CE, Piamjariyakul U, Dalton KM, Russell C, Wick J, Ellerbeck EF. Nurse-led 
multidisciplinary heart failure group clinic appointments: methods, materials and outcomes used in the clinical trial. J Cardiovasc Nurs. 2015; 30(4):25-34.

28. Bala SV, Samuelsson K, Hagell P, Svensson $B$, Fridlund B, Hesselgard K. The experience of care at nurse-led rheumatology clinics. Musculoskeletal Care. 2012; 10(4):202-11.

29. Ferguson $\mathrm{C}$, Inglis SC, Newton, PJ, Middleton S, Macdonald PS, Davidson PM. Education and practice gaps on atrial fibrillation and anticoagulation: a survey of cardiovascular nurses. BMC Med Educ. 2016; 16(1):9-18.

30. Jesus MCP, Figueiredo MAG, Santos SMR, Amaral AMM, Rocha LO, Thiollent MJM. Educação permanente em enfermagem em um hospital universitário. Rev Esc Enf USP. 2011; 45(5): 1229-36.

31. Bessa ATTD, Mesquita MGDR, Stipp MAC.
Saúde do homem e doença cardiovascular: gerenciamento do cuidado de enfermagem em nível ambulatorial. Rev Pesqui Cuid Fundam. [Internet]. 2016 [citado em 27 out 2017]; 8(3):4766-72. Disponível em: https://www.redalyc.org/pdf/5057/505754 106017.pdf DOI: http://www.redalyc.org/articulo.oa?id=5057 54106017

\section{CONTRIBUIÇÕES}

Diego da Silva Moreira foi responsável pela concepção do projeto, delineamento, análise, interpretação dos dados e redação. Priscila Cristina da Silva Thiengo contribuiu na análise, interpretação dos dados e revisão. Célia Caldeira Fonseca Kestenberg orientou todas as etapas do trabalho e participou da revisão. Elizabeth Rose Costa Martins e Alexandre Vicente da Silva participaram da revisão crítica.

\footnotetext{
Como citar este artigo (Vancouver)

Moreira DS, Kestenberg CCF, Thiengo PCS, Silva AV, Martins ERC. Habilidades empáticas na consulta de enfermagem ao cliente com doença cardiovascular: uma revisão integrativa. REFACS [Internet]. 2019 [citado em inserir dia, mês e ano de acesso]; 7(2):227-239. Disponível em: inserir link de acesso. DOI: inserir link do DOI.

\section{Como citar este artigo (ABNT)}

MOREIRA, D. S.; KESTENBERG, C. C. F.; THIENGO, P. C. S.; SILVA, A. V.; Martins, E. R. C. Habilidades empáticas na consulta de enfermagem ao cliente com doença cardiovascular: uma revisão integrativa. REFACS, Uberaba, MG, v. 7, n. 2, p. 227-239, 2019. DOI: inserir link do DOI. Disponível em: inserir link de acesso. Acesso em: inserir dia, mês e ano de acesso.

\section{Como citar este artigo (APA)}

Moreira, D.S., Kestenberg, C.C.F., Thiengo, P.C.S., Silva, A.V., Martins, E.R.C. Habilidades empáticas na consulta de enfermagem ao cliente com doença cardiovascular: uma revisão integrativa. REFACS, 7(2), 227-239. Recuperado em: inserir dia, mês e ano de acesso de inserir link de acesso. DOI: inserir link do DOI.
} 